\title{
Du côté de la Cité des sciences et de l'industrie
}

Nouvelles technologies et action éducative

Jean-Marie Sani

\section{CpenEdition}

Journals

Édition électronique

URL : http://journals.openedition.org/ries/2903

DOI : 10.4000/ries.2903

ISSN : 2261-4265

Éditeur

Centre international d'études pédagogiques

Édition imprimée

Date de publication : 1 juin 1998

Pagination : 55-58

ISSN : $1254-4590$

Référence électronique

Jean-Marie Sani, «Du côté de la Cité des sciences et de l'industrie », Revue internationale d'éducation de Sèvres [En ligne], 18 | juin 1998, mis en ligne le 18 avril 2013, consulté le 22 avril 2019. URL : http:// journals.openedition.org/ries/2903 ; DOI : 10.4000/ries.2903

Ce document a été généré automatiquement le 22 avril 2019

(c) Tous droits réservés 


\title{
Du côté de la Cité des sciences et de l'industrie
}

\author{
Nouvelles technologies et action éducative
}

Jean-Marie Sani

1 La Cité des sciences et de l'industrie a pour mission de rendre le développement des sciences, des techniques et du savoir-faire industriel perceptible et compréhensible par tous les publics qu'elle peut atteindre, soit directement (visiteurs), soit indirectement dans les régions ou à l'étranger. La mission de l'établissement est du reste définie dans l'article 2 du décret de 1985 qui l'a créé. On pourrait dire, en somme, que la Cité des sciences a pour fonction de montrer, d'expliquer, de faire comprendre les évolutions en cours et de susciter, chez les visiteurs, une réflexion sur les conséquences prévisibles de celles-ci dans la société et dans la vie quotidienne.

2 Parmi les domaines qui ont précisément de nombreuses incidences sur la société, les nouvelles technologies de l'information et de la communication (NTIC) constituent pour la Cité à la fois un sujet et un média.

3 C'est d'abord un sujet, car les transformations qui sont induites par le développement de ces technologies sont nombreuses: l'information, la communication, le commerce, les services, le fonctionnement des entreprises, le rapport à l'administration sont autant de champs qui sont ou seront modifiés par leur développement. C'est pourquoi, nous avons souhaité proposer au public une exposition temporaire: depuis mi-décembre 1997, "Nouvelle image, nouveaux réseaux » aborde une partie de ces aspects et son succès public a confirmé l'intérêt de nos concitoyens pour ceux-ci.

4 C'est aussi un média. Toute institution dont la vocation est de communiquer s'interroge sur les bouleversements que ces technologies vont provoquer sur son activité. Les musées s'y trouvent confrontés de manière particulière puisqu'ils sont traditionnellement des lieux géographiquement déterminés où sont présentés des objets physiques. Pourtant, un deuxième type de musée apparaît : ce sont les musées virtuels qui, à distance, peuvent aussi jouer un rôle et donner à voir, à comprendre et à connaître, par écran interposé, à ce qu'il convient désormais d'appeler son « cyber-visiteur ». La Cité s'est donc engagée dans le développement d'un site «Web» qui va offrir des services croissants de mois en 
mois. Elle a, par ailleurs, produit une plate-forme de réseau à haut débit qui, d'une part, préfigure les réseaux du futur et, d'autre part, devient un outil de production et de diffusion de ses contenus.

5 En matière d'éducation, nous nous devions d'apporter notre contribution. Les nouvelles technologies peuvent être alors considérées de trois manières :

- les réseaux, le micro-ordinateur, les logiciels, le multimédia sont des objets techniques qu'il convient de maîtriser et leur pratique est une fin en soi ;

- les technologies constituent un outil de communication et de téléenseignement; les techniques de visioconférence, de documents partagés, de travail coopératif à distance sont des outils en développement essentiels à cet aspect : on pourra ainsi accéder à des options rares, désenclaver des régions, favoriser le développement de certains pays ;

- les NTIC constituent un outil pour la pratique de la classe, au même titre que le manuel, l'audiovisuel et ce pour toutes les disciplines, des lettres aux sciences exactes, en passant par les arts plastiques et les langues.

6 C'est particulièrement ce troisième axe que nous avons étudié et expérimenté avec des classes et des groupes d'enseignants. Dans ce cadre, il nous est apparu que l'usage de ces outils au même titre que d'autres, plus traditionnels dans la classe, pouvait être lui-même subdivisé en trois catégories :

- la recherche d'informations; la ressource disponible étant considérable, que ce soit sur Internet ou sur des cédéroms de type encyclopédique, le premier savoir-faire à acquérir concerne la recherche documentaire. Ceci exige une bonne formulation des questions, une méthode de recherche de sources d'information, le traitement de ces ressources et l'exercice de l'esprit critique quant à la qualité de cette information ;

- la rédaction et la synthèse ; les outils de la bureautique permettent d'effectuer des exercices de rédaction et de synthèse, simplement, tout d'abord avec un traitement de texte. La forme du document peut être plus riche en terme de média si l'on utilise un des éditeurs de pages HTML disponible; ces outils se simplifient de plus en plus aujourd'hui. Après l'activité de recherche, la synthèse et la rédaction permettent la reconstruction et la reformulation nécessaires à l'acquisition de connaissances et à la qualité de la communication ;

- la communication ; c'est la troisième modalité. L'échange de correspondances, qui peut dès lors être multimédia, et la collaboration pour produire un document sont possibles. Ceci permet la constitution d'un corpus de connaissances issu d'environnements locaux divers, les échanges interculturels, les apprentissages linguistiques.

7 Ces trois modalités ne sont pas la conséquence de l'apparition des NTIC : la recherche documentaire est une technique ancienne et les centres de documentation dans les établissements scolaires y initient les élèves ; la recherche d'information se pratique dans des environnements variés, lors de visites d'un milieu naturel ou d'un musée. La réalisation de synthèses est aussi une pratique ancienne : la dissertation en est une des formes, comme l'exposé, l'exposition d'école. La souris remplace les ciseaux et la colle, le résultat est différent, mais la démarche est la même. Enfin, la correspondance scolaire existe depuis longtemps, surtout à l'école primaire. On n'utilise plus le courrier postal mais les e-mail. On voit que ces usages ne sont pas nouveaux. Il est utile de pratiquer les outils que nous proposent les NTIC, pour les maitriser.

Dès 1994, la Cité des sciences et de l'industrie s'est engagée dans une réflexion qui conduit à la mise en place de programmes valorisant l'usage des NTIC en général, dans l'éducation en particulier. Nous nous sommes ainsi engagés dans l'organisation de «Cliquez pour le Savoir ! ». Cette exposition-formation qui a eu lieu deux fois, en mars 1995 et en octobre 
1996, permettait au public, enseignant en particulier, de pratiquer les outils du multimédia et de s'en faire une opinion. Il s'agissait donc de proposer une sélection de cédéroms disponibles sur le marché, couvrant de manière exemplaire le champ du ludoéducatif et du culturel. Une centaine de ces produits était présentée à chaque édition, des ateliers permettaient d'approfondir les pratiques techniques ou pédagogiques. Un troisième espace proposait un programme quotidien où l'on pouvait prendre connaissance d'innovations technologiques, de projets pédagogiques, de l'action d'organismes ou d'associations. Durant ces deux manifestations, la fonction d'initiation pour un large public était assumée. C'était notre objectif, nous avions écarté le principe de l'organisation d'un événement uniquement destiné aux aficionados. Lors de la deuxième édition, l'offre des réseaux était présentée, comme complément de l'offre hors ligne. Enfin, nous avons maintenant le projet d'organiser une troisième édition de «Cliquez pour le Savoir ! » dans le courant de l'an 2000.

En mars 1998, les XXI ${ }^{e}$ Entretiens de la Villette étaient consacrés au thème des "Multimédia et Réseaux ». À travers conférences, ateliers, visites d'expositions, nous avons proposé au public enseignant de s'informer et de se former sur ce sujet, en traitant d'aspects scientifiques et techniques, des enjeux sociaux et des pratiques éducatives. Cette manifestation était pour la première fois simultanément conduite dans trois sites régionaux (Nice, Chambéry et Montpellier) avec un certain nombre de conférences ayant simultanément lieu dans les quatre sites par le biais de visioconférences. Par ailleurs, nous avons organisé une quatrième journée, en direction du grand public, principalement de parents d'élèves. Enfin, comme il est dit plus haut, la grande exposition temporaire de l'année 1998, « Nouvelle image, nouveaux réseaux », traite de ces questions.

Notre action éducative s'appuie sur une réflexion préalable relative aux contenus, sur la mise en œuvre d'expériences concrètes, sur une formalisation de ces pratiques et des conclusions que l'on peut en tirer. Cette action, pour tous les thèmes que nous nous donnons, ne peut s'appuyer uniquement sur une stratégie d'événement. Il convient par ailleurs de conduire une action dans la durée, en permanence. Il y a maintenant un an, nous avons mis en place une salle multimédia. Il s'agit d'un réseau Ethernet de dix ordinateurs PC, doté de trois imprimantes, d'un banc de montage vidéo numérique, d'un scanner, d'un accès à Internet, d'appareils photos numériques, chaque terminal ayant par ailleurs une caméra. Cette plate-forme polyvalente a pour fonction de tester des pratiques d'enseignement et de médiation. Différents services de la Cité y ont accès : l'animation, qui a pu préparer l'exploitation de l'exposition « Nouvelle image, nouveaux réseaux », la cité des enfants, la cité des métiers dont le cercle de recherche d'emplois accède à Internet pour parcourir les petites annonces, mais aussi et surtout pour mieux connaître les entreprises et d'éventuels employeurs. Cet équipement est aussi utilisé à des fins de formation interne.

En ce qui concerne l'action éducative, plusieurs activités s'y sont associées :

- la classe Villette Environnement y effectue des recherches d'information sur des sites de collectivités locales et y pratique un jeu de « Simcity $^{\circledR}$ » en réseau, à la suite duquel un travail peut être accompli sur l'importance de l'administration municipale ;

- la classe Villette Communication y réalise un exercice d'animation de figurines comme à l'aide d'un banc titre de cinéma ;

- une classe Villette spécifique de terminale "action commerciale et administrative » a travaillé, en janvier 1998, d'une part sur les techniques de communication, d'autre part sur la conception et la réalisation d'une page Web ; 
- une classe Villette spécifique de $4^{\mathrm{e}}$ en ZEP à Soissons. Le projet de cette classe était de créer un document multimédia sur les innovations technologiques du $\mathrm{XX}^{\mathrm{e}}$ siècle. Quatre groupes travaillaient sur différents thèmes (espace, informatique, santé, automobile). Les élèves avaient préalablement produit des textes; à la Cité, ils ont accumulé de l'information, des images, enregistrements audio et documents et ont commencé la réalisation des pages HTML. Ce travail, a abouti à la production d'un cédérom dont le contenu pourra être rendu public sur un site Web académique.

Pour les mois qui viennent, nous allons bien entendu poursuivre ces actions. Parmi les domaines que nous souhaitons approfondir, nous pouvons citer :

- la formation des enseignants ;

- le développement de la partie du site Web de la Cité qui leur est destinée ;

- le développement du travail coopératif à distance.

L'utilisation dans l'éducation des NTIC constitue une des priorités de l'action éducative de la Cité des sciences et de l'industrie. Nous nous efforçons de participer à ce vaste mouvement que l'on observe dans la plupart des systèmes éducatifs pour montrer que cet outil, sans remplacer les autres, peut et doit devenir un des moyens importants de travail et d'ouverture au monde pour les élèves.

\section{RÉSUMÉS}

L'utilisation des nouvelles technologies dans l'éducation est une priorité de l'action éducative de la Cité des sciences et de l'industrie. Expos-formation, conférences et visio-conférences, ateliers complètent la panoplie des classes Villette et des actions de formation des enseignants

\section{INDEX}

Index géographique : France

Mots-clés : cité des sciences et de l'industrie, éducation scientifique, nouvelles technologies

\section{AUTEUR}

JEAN-MARIE SANI

Chef du département Éducation, Cité des sciences et de l'industrie, Paris, France 